Acta Crystallographica Section E

Structure Reports

Online

ISSN 1600-5368

\section{O,O-Bis(2-tert-butyl-4-methoxyphenyl) chlorothiophosphonate. Corrigendum.}

In the original report by Odabaşoğlu, Büyükgüngör \& Albayrak [Acta Cryst. (2005), E61, o2528-o2530], the structure was reported in the incorrect space group $C c$. The structure is now reported as disordered in the correct space group $C 2 / c$. The $\mathrm{P}$ atom lies on a twofold rotation axis. A revised description of the hydrogen bonding is also given. University, TR-55139 Samsun, Turkey, and ${ }^{\mathbf{b}}$ Department of Physics, Ondokuz Mayıs

University, TR-55139 Samsun, Turkey

Correspondence e-mail: orhanb@omu.edu.tr

\section{Key indicators}

Single-crystal X-ray study

$T=150 \mathrm{~K}$

Mean $\sigma(\mathrm{C}-\mathrm{C})=0.002 \AA$

Disorder in main residue

$R$ factor $=0.028$

$w R$ factor $=0.073$

Data-to-parameter ratio $=12.1$

For details of how these key indicators were automatically derived from the article, see http://journals.iucr.org/e.
(C) 2005 International Union of Crystallography Printed in Great Britain - all rights reserved

\section{Comment}

An ORTEP-3 (Farrugia, 1997) view of (I) and a packing diagram are shown in Figs. 1 and 2, respectively. The $\mathrm{P}$ atom lies on a twofold rotation axis, eading to disorder of the $\mathrm{Cl}$ and $\mathrm{S}$ atoms. Compound (I) has no classical hydrogen bonds, but there are two $\mathrm{C}-\mathrm{H} \cdots \pi$ interactions: $\mathrm{H} 8 A \cdots C g 1=3.12(2) \AA$ and $\mathrm{C} 8-\mathrm{H} 8 A \cdots C g 1=122.8(2)^{\circ}(C g 1$ is the centroid of the $\mathrm{C}^{\mathrm{ii}}-\mathrm{C} 6^{\mathrm{ii}}$ ring), and $\mathrm{H} 11 A \cdots C g 2=2.71(2) \AA$ and $\mathrm{C} 11-$ $\mathrm{H} 11 A \cdots C g 2=142.7(1)^{\circ}\left(C g 2\right.$ is the centroid of the $\mathrm{C}^{\mathrm{iii}}-\mathrm{C}^{\mathrm{iii}}$

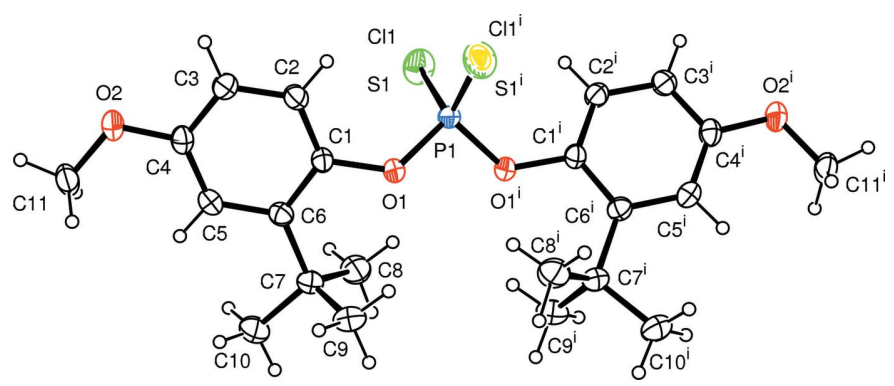

Figure 1

A view of (I), with the atom-numbering scheme and $50 \%$ probability displacement ellipsoids.[Symmetry code: (i) $-x+1, y,-z+\frac{1}{2}$ ]

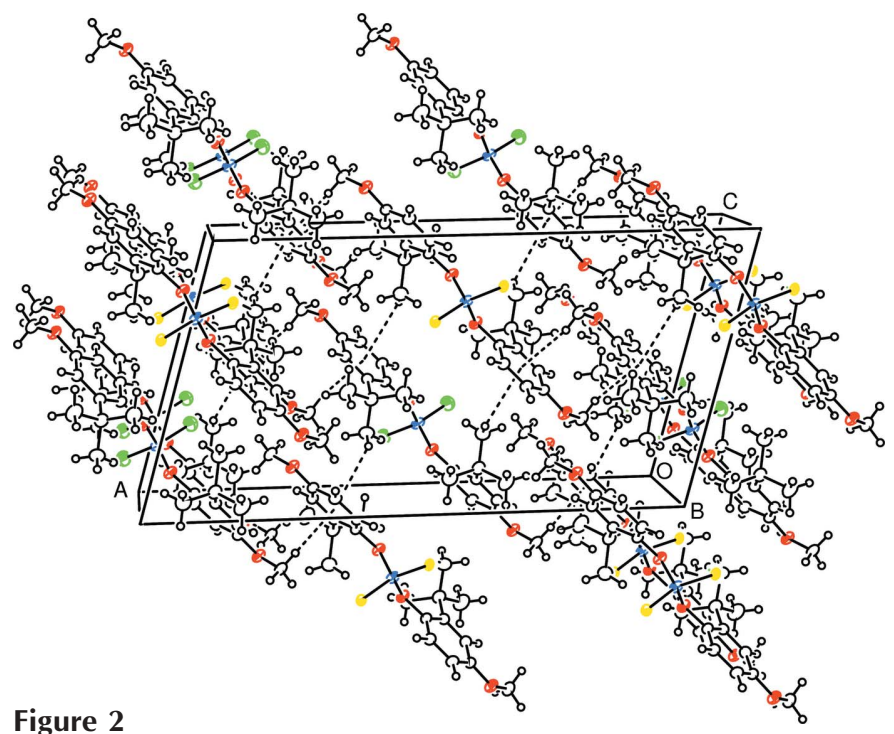

A view of the packing of (I); $\mathrm{C}-\mathrm{H} \cdots \pi$ interactions are drawn as dashed lines.
Received 8 August 2005 Accepted 5 September 2005 Online 17 September 2005 
ring) [symmetry codes: (ii) $x, 1-y, \frac{1}{2}+z$; (iii) $\left.\frac{1}{2}-x, \frac{1}{2}-y,-z\right]$. The dihedral angle between the symmetry-related benzene rings is $41.2(2)^{\circ}$. Selected bond distances and angles are given in Table 1.

\section{Experimental}

\section{Crystal data}

\section{$\mathrm{C}_{22} \mathrm{H}_{30} \mathrm{ClO}_{4} \mathrm{PS}$}

$M_{r}=456.94$

Monoclinic, $C 2 / c$

$a=23.592(3) \AA$

$b=8.3111(6) \AA$

$c=12.5067$ (14) $\AA$

$\beta=105.740(9)^{\circ}$

$V=2360.3(4) \AA^{3}$

$Z=4$

\section{Data collection}

Stoe IPDS-II diffractometer $\omega$ scans

Absorption correction: integration

( $X$-RED32; Stoe \& Cie, 2002)

$T_{\text {min }}=0.824, T_{\text {max }}=0.869$

16485 measured reflections

2327 independent reflections

\section{Refinement}

Refinement on $F^{2}$

$R\left[F^{2}>2 \sigma\left(F^{2}\right)\right]=0.028$

$w R\left(F^{2}\right)=0.073$

$S=1.08$

2327 reflections

192 parameters

All $\mathrm{H}$-atom parameters refined

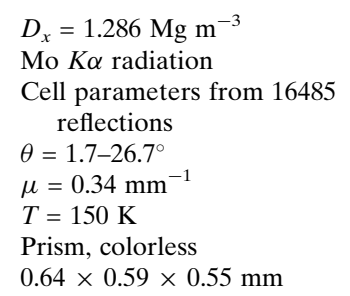

2146 reflections with $I>2 \sigma(I)$

$R_{\text {int }}=0.032$

$\theta_{\max }=26.0^{\circ}$

$h=-28 \rightarrow 28$

$k=-10 \rightarrow 10$

$l=-15 \rightarrow 15$

$$
\begin{gathered}
w=1 /\left[\sigma^{2}\left(F_{\mathrm{o}}{ }^{2}\right)+(0.0362 P)^{2}\right. \\
+1.6163 P] \\
\text { where } P=\left(F_{\mathrm{o}}^{2}+2 F_{\mathrm{c}}^{2}\right) / 3 \\
(\Delta / \sigma)_{\max }=0.002 \\
\Delta \rho_{\max }=0.17 \mathrm{e}^{-3} \\
\Delta \rho_{\min }=-0.34 \mathrm{e}^{-3}
\end{gathered}
$$

Table 1

Selected geometric parameters $\left(\AA{ }^{\circ}{ }^{\circ}\right)$.

\begin{tabular}{llll}
\hline $\mathrm{C} 1-\mathrm{C} 2$ & $1.3946(18)$ & $\mathrm{C} 4-\mathrm{C} 5$ & $1.4017(19)$ \\
$\mathrm{C} 1-\mathrm{C} 6$ & $1.4086(17)$ & $\mathrm{C} 5-\mathrm{C} 6$ & $1.4088(18)$ \\
$\mathrm{C} 1-\mathrm{O} 1$ & $1.4226(14)$ & $\mathrm{O} 1-\mathrm{P} 1$ & $1.5750(9)$ \\
$\mathrm{C} 2-\mathrm{C} 3$ & $1.3860(18)$ & $\mathrm{P} 1-\mathrm{S} 1^{\mathrm{i}}$ & $1.9791(4)$ \\
$\mathrm{C} 3-\mathrm{C} 4$ & $1.3979(18)$ & $\mathrm{P} 1-\mathrm{Cl} 1$ & $1.9791(4)$ \\
& & & \\
$\mathrm{C} 2-\mathrm{C} 1-\mathrm{C} 6$ & $123.25(11)$ & $\mathrm{C} 4-\mathrm{C} 5-\mathrm{C} 6$ & $121.99(12)$ \\
$\mathrm{C} 2-\mathrm{C} 1-\mathrm{O} 1$ & $119.13(11)$ & $\mathrm{C} 1-\mathrm{C} 6-\mathrm{C} 5$ & $115.48(11)$ \\
$\mathrm{C} 6-\mathrm{C} 1-\mathrm{O} 1$ & $117.58(11)$ & $\mathrm{O} 1-\mathrm{P} 1-\mathrm{O} 1^{\mathrm{i}}$ & $97.08(7)$ \\
$\mathrm{C} 3-\mathrm{C} 2-\mathrm{C} 1$ & $119.77(12)$ & $\mathrm{O} 1-\mathrm{P} 1-\mathrm{S} 1^{\mathrm{i}}$ & $111.27(4)$ \\
$\mathrm{C} 2-\mathrm{C} 3-\mathrm{C} 4$ & $119.11(12)$ & $\mathrm{O} 1-\mathrm{P} 1-\mathrm{Cl} 1$ & $111.44(3)$ \\
$\mathrm{C} 3-\mathrm{C} 4-\mathrm{C} 5$ & $120.39(11)$ & $\mathrm{S} 1^{\mathrm{i}}-\mathrm{P} 1-\mathrm{Cl} 1$ & $113.26(3)$ \\
\hline
\end{tabular}

Symmetry code: (i) $-x+1, y,-z+\frac{1}{2}$.

All $\mathrm{H}$ atoms were refined freely. Atoms $\mathrm{S} 1$ and $\mathrm{Cl} 1$ were assigned to the same atomic site and refined freely with the same atomic coordinates and with fixed site-occupancy factors of 0.5 .

Data collection: $X$-AREA (Stoe \& Cie, 2002); cell refinement: $X$-AREA; data reduction: $X$-RED32 (Stoe \& Cie, 2002); program(s) used to solve structure: SHELXS97 (Sheldrick, 1997); program(s) used to refine structure: SHELXL97 (Sheldrick, 1997); molecular graphics: ORTEP-3 for Windows (Farrugia, 1997); software used to prepare material for publication: Win $G X$ (Farrugia, 1999).

\section{References}

Farrugia, L. J. (1997). J. Appl. Cryst. 30, 565.

Farrugia, L. J. (1999). J. Appl. Cryst. 32, 837-838.

Sheldrick, G. M. (1997). SHELXS97 and SHELXL97. University of Göttingen, Germany.

Stoe \& Cie (2002). X-AREA (Version 1.18) and X-RED32 (Version 1.04). Stoe \& Cie, Darmstadt, Germany. 


\section{supporting information}

Acta Cryst. (2005). E61, e4-e5 [doi:10.1107/S1600536805027789]

\section{O,O-Bis(2-tert-butyl-4-methoxyphenyl) chlorothiophosphonate. Corrigendum.}

\section{Mustafa Odabaşoğlu, Orhan Büyükgüngör and Çiğdem Albayrak}

\section{S1. Comment}

An ORTEP-3 (Farrugia, 1997) view of (I) and a packing diagram are shown in Figs. 1 and 2, respectively. Compound (I) has no classical hydrogen bonds but there are two $\mathrm{C}-\mathrm{H} \cdots \pi$ interactions: $\mathrm{H} 8 A \cdots C g 1=3.12(2) \AA$ and $\mathrm{C} 8-\mathrm{H} 8 A \cdots C g 1=$ $122.8(2)^{\circ}\left[C g 1\right.$ is the centroid of the $\mathrm{C} 1^{\mathrm{i}}-\mathrm{C} 6^{\mathrm{i}}$ ring; and $\mathrm{H} 11 A \cdots C g 2=2.71(2) \AA$ and $\mathrm{C} 11-\mathrm{H} 11 A \cdots C g 2=142.7(1)^{\circ}$ ( $C g 2$ is the centroid of the $\mathrm{C}{ }^{\mathrm{ii}} \_\mathrm{C} 6^{\mathrm{ii}}$ ring); symmetry codes: (i) $x, 1-y, 1 / 2+z$; (ii) $\left.1 / 2-x, 1 / 2-y,-z\right]$. The dihedral angle between the symmetry-related benzene rings in (I) is $41.2(2)^{\circ}$. Selected bond distances and angles for (I) are given in Table 1. The benzene ring is planar, with a maximum deviation from the plane defined by the six ring atoms of 0.007 (1) A (for C3).

\section{S2. Experimental}

Compound (I) was prepared by the method of Odabaşoğlu et al. (2005), using 2-tert-butyl-4-methoxyphenol and $\mathrm{PSCl}_{3}$ as starting materials. Crystals of (I) suitable for single-crystal X-ray diffraction were grown by slow evaporation of a solution in $n$-hexane (yield 77\%, m.p. $423 \mathrm{~K}$ ). Analysis calculated: C 57.83, H 6.57\%; found: C 57.36, H 6.38\%.

\section{S3. Refinement}

All $\mathrm{H}$ atoms were refined freely. Atoms S1 and Cl1 were assigned to the same atomic site and refined freely with the same atomic coordinates and with fixed site-occupancy factors of 0.5 .

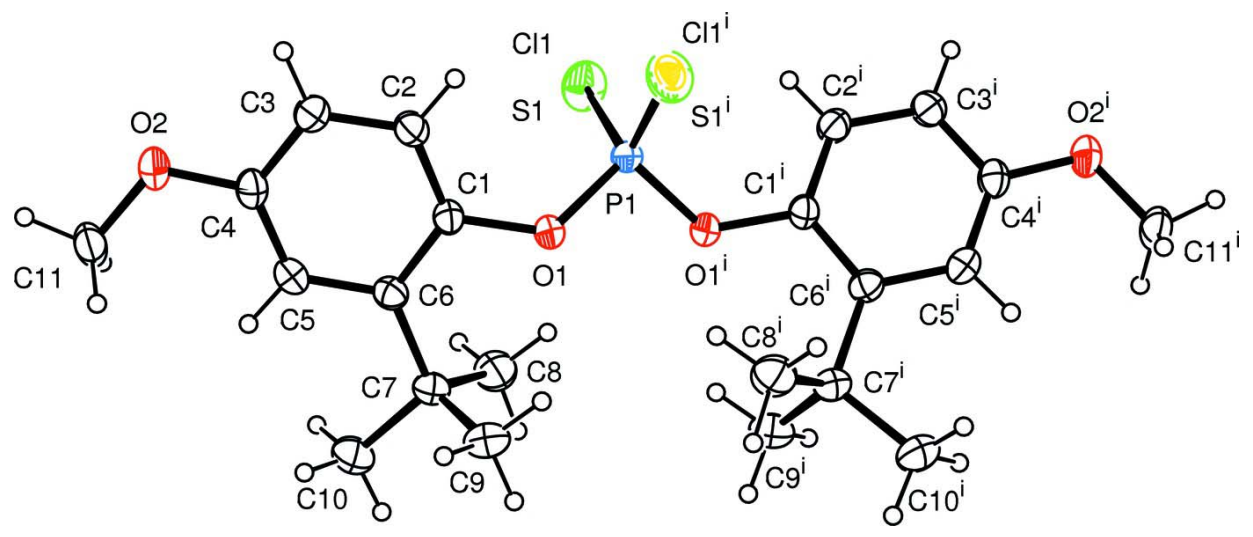

\section{Figure 1}

A view of (I), with the atom-numbering scheme and 50\% probability displacement ellipsoids·[Symmetry codes: (i) $-x, y$, $-z+1 / 2]$ 


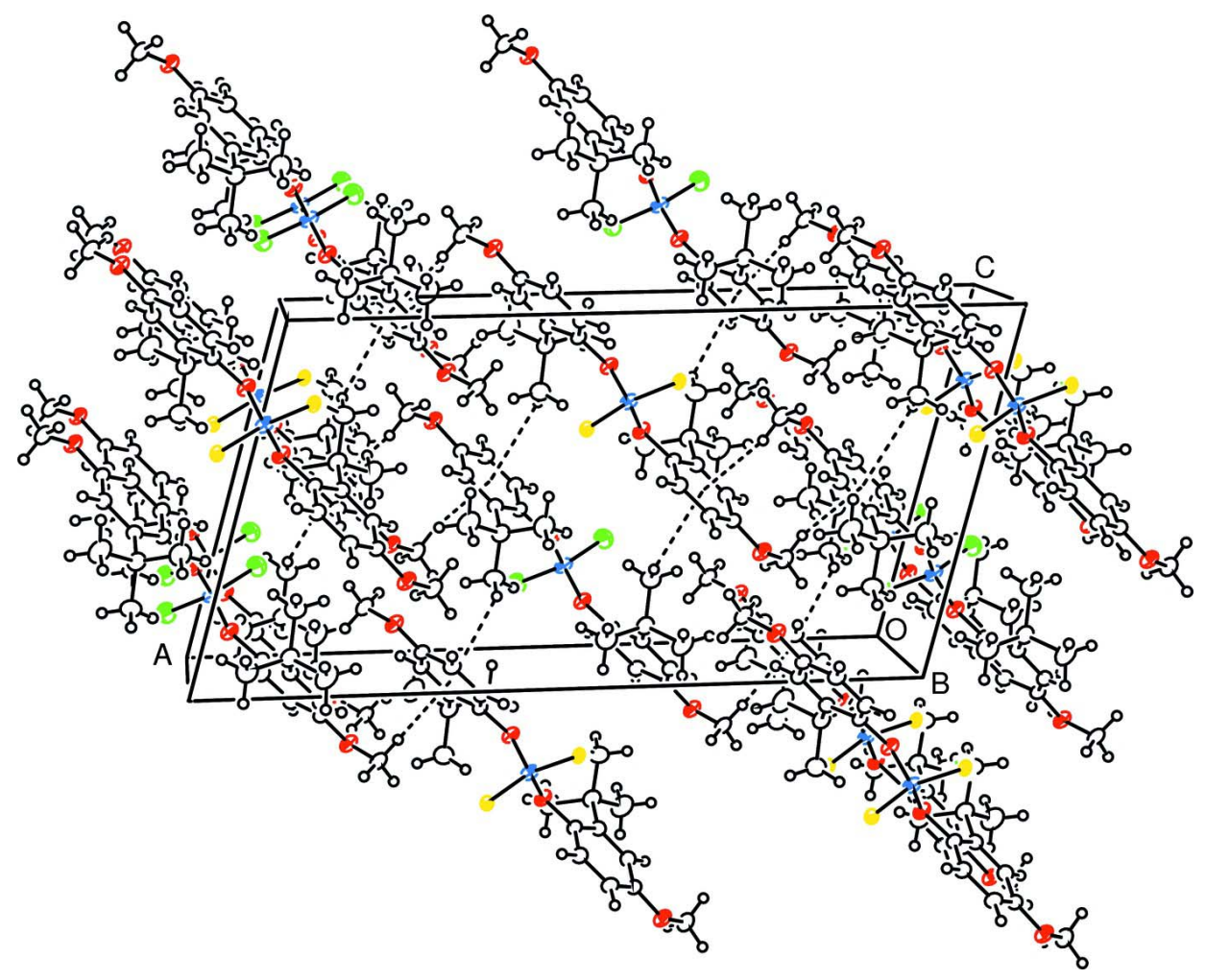

Figure 2

A view of the packing of (I); $\mathrm{C}-\mathrm{H} \cdots \pi$ interactions are drawn as dashed lines.

\section{O,O-Bis(2-tert-butyl-4-methoxyphenyl) chlorothiophosphonate}

Crystal data

$\mathrm{C}_{22} \mathrm{H}_{30} \mathrm{ClO}_{4} \mathrm{PS}$

$M_{r}=456.94$

Monoclinic, $C 2 / c$

Hall symbol: $-\mathrm{C} 2 \mathrm{yc}$

$a=23.592(3) \AA$

$b=8.3111(6) \AA$

$c=12.5067(14) \AA$

$\beta=105.740(9)^{\circ}$

$V=2360.3(4) \AA^{3}$

$Z=4$

\section{Data collection}

Stoe IPDS-II

diffractometer

Graphite monochromator

$\omega$ scans

Absorption correction: integration

(X-RED32; Stoe \& Cie, 2002)

$T_{\min }=0.824, T_{\max }=0.869$

16485 measured reflections
$F(000)=968$

$D_{\mathrm{x}}=1.286 \mathrm{Mg} \mathrm{m}^{-3}$

Mo $K \alpha$ radiation, $\lambda=0.71073 \AA$

Cell parameters from 16485 reflections

$\theta=1.7-26.7^{\circ}$

$\mu=0.34 \mathrm{~mm}^{-1}$

$T=150 \mathrm{~K}$

Prism, colorless

$0.64 \times 0.59 \times 0.55 \mathrm{~mm}$

2327 independent reflections

2146 reflections with $I>2 \sigma(I)$

$R_{\text {int }}=0.032$

$\theta_{\text {max }}=26.0^{\circ}, \theta_{\text {min }}=1.8^{\circ}$

$h=-28 \rightarrow 28$

$k=-10 \rightarrow 10$

$l=-15 \rightarrow 15$ 


\section{Refinement}

Refinement on $F^{2}$

Least-squares matrix: full

$R\left[F^{2}>2 \sigma\left(F^{2}\right)\right]=0.028$

$w R\left(F^{2}\right)=0.073$

$S=1.08$

2327 reflections

192 parameters

0 restraints

Primary atom site location: structure-invariant direct methods
Secondary atom site location: difference Fourier map

Hydrogen site location: difference Fourier map

All H-atom parameters refined

$w=1 /\left[\sigma^{2}\left(F_{\mathrm{o}}^{2}\right)+(0.0362 P)^{2}+1.6163 P\right]$

where $P=\left(F_{\mathrm{o}}^{2}+2 F_{\mathrm{c}}^{2}\right) / 3$

$(\Delta / \sigma)_{\max }=0.002$

$\Delta \rho_{\max }=0.17 \mathrm{e} \AA^{-3}$

$\Delta \rho_{\text {min }}=-0.34$ e $\AA^{-3}$

Special details

Geometry. All e.s.d.'s (except the e.s.d. in the dihedral angle between two 1.s. planes) are estimated using the full covariance matrix. The cell e.s.d.'s are taken into account individually in the estimation of e.s.d.'s in distances, angles and torsion angles; correlations between e.s.d.'s in cell parameters are only used when they are defined by crystal symmetry. An approximate (isotropic) treatment of cell e.s.d.'s is used for estimating e.s.d.'s involving 1.s. planes.

Refinement. Refinement of $F^{2}$ against ALL reflections. The weighted $R$-factor $w R$ and goodness of fit $S$ are based on $F^{2}$, conventional $R$-factors $R$ are based on $F$, with $F$ set to zero for negative $F^{2}$. The threshold expression of $F^{2}>\sigma\left(F^{2}\right)$ is used only for calculating $R$-factors(gt) etc. and is not relevant to the choice of reflections for refinement. $R$-factors based on $F^{2}$ are statistically about twice as large as those based on $F$, and $R$ - factors based on ALL data will be even larger.

Fractional atomic coordinates and isotropic or equivalent isotropic displacement parameters $\left(\AA^{2}\right)$

\begin{tabular}{|c|c|c|c|c|c|}
\hline & $x$ & $y$ & $z$ & $U_{\text {iso }} * / U_{\text {eq }}$ & Occ. $(<1)$ \\
\hline $\mathrm{C} 1$ & $0.58872(5)$ & $0.29212(15)$ & $0.41994(10)$ & $0.0224(3)$ & \\
\hline $\mathrm{C} 2$ & $0.59796(6)$ & $0.14466(16)$ & $0.47516(11)$ & $0.0258(3)$ & \\
\hline $\mathrm{C} 3$ & $0.65010(6)$ & $0.11722(16)$ & $0.55649(11)$ & $0.0272(3)$ & \\
\hline $\mathrm{C} 4$ & $0.69210(5)$ & $0.23993(16)$ & $0.58337(10)$ & 0.0249 (3) & \\
\hline $\mathrm{C} 5$ & $0.68201(6)$ & $0.38769(16)$ & $0.52737(11)$ & $0.0252(3)$ & \\
\hline C6 & $0.62988(5)$ & $0.41888(15)$ & $0.44326(10)$ & 0.0233 & \\
\hline $\mathrm{C} 7$ & $0.61903(6)$ & $0.58369(16)$ & $0.38318(12)$ & $0.0296(3)$ & \\
\hline $\mathrm{C} 8$ & $0.61032(8)$ & $0.56105(19)$ & $0.25687(13)$ & $0.0374(3)$ & \\
\hline C9 & $0.56458(8)$ & $0.66639(19)$ & $0.40646(15)$ & $0.0402(4)$ & \\
\hline $\mathrm{C} 10$ & $0.67218(8)$ & $0.6983(2)$ & $0.42394(16)$ & $0.0453(4)$ & \\
\hline $\mathrm{C} 11$ & $0.78481(6)$ & $0.32952(19)$ & $0.70159(13)$ & $0.0343(3)$ & \\
\hline O1 & $0.53449(4)$ & $0.31975(10)$ & $0.33826(7)$ & $0.0257(2)$ & \\
\hline $\mathrm{O} 2$ & $0.74184(4)$ & $0.20383(12)$ & $0.66690(8)$ & $0.0319(2)$ & \\
\hline P1 & 0.5000 & $0.19428(5)$ & 0.2500 & $0.02610(13)$ & \\
\hline $\mathrm{Cl1}$ & $0.554354(14)$ & $0.06331(4)$ & $0.18992(3)$ & $0.03162(11)$ & 0.50 \\
\hline S1 & $0.554354(14)$ & $0.06331(4)$ & $0.18992(3)$ & $0.03162(11)$ & 0.50 \\
\hline $\mathrm{H} 2$ & $0.5683(7)$ & $0.0634(19)$ & $0.4571(13)$ & $0.032(4)^{*}$ & \\
\hline $\mathrm{H} 3$ & $0.6579(7)$ & $0.014(2)$ & $0.5947(13)$ & $0.034(4)^{*}$ & \\
\hline H5 & $0.7120(7)$ & $0.4671(19)$ & $0.5482(13)$ & $0.029(4)^{*}$ & \\
\hline $\mathrm{H} 8 \mathrm{~A}$ & $0.6047(8)$ & $0.668(3)$ & $0.2193(16)$ & $0.052(5)^{*}$ & \\
\hline H8B & $0.5774(8)$ & $0.495(2)$ & $0.2241(15)$ & $0.043(5)^{*}$ & \\
\hline $\mathrm{H} 8 \mathrm{C}$ & $0.6466(9)$ & $0.512(2)$ & $0.2429(17)$ & $0.056(5)^{*}$ & \\
\hline H9A & $0.5571(8)$ & $0.772(2)$ & $0.3671(15)$ & $0.051(5)^{*}$ & \\
\hline H9B & $0.5703(8)$ & $0.686(2)$ & $0.4892(18)$ & $0.056(5)^{*}$ & \\
\hline $\mathrm{H} 9 \mathrm{C}$ & $0.5281(8)$ & $0.601(2)$ & $0.3804(15)$ & $0.047(5)^{*}$ & \\
\hline
\end{tabular}




\begin{tabular}{lllll} 
H10A & $0.7097(9)$ & $0.650(2)$ & $0.4105(16)$ & $0.056(6)^{*}$ \\
H10B & $0.6816(9)$ & $0.720(2)$ & $0.5075(19)$ & $0.059(6)^{*}$ \\
H10C & $0.6631(9)$ & $0.798(2)$ & $0.3852(17)$ & $0.056(5)^{*}$ \\
H11A & $0.8030(8)$ & $0.360(2)$ & $0.6410(15)$ & $0.042(5)^{*}$ \\
H11B & $0.8151(8)$ & $0.282(2)$ & $0.7644(15)$ & $0.041(4)^{*}$ \\
H11C & $0.7671(7)$ & $0.427(2)$ & $0.7281(14)$ & $0.035(4)^{*}$ \\
\hline
\end{tabular}

Atomic displacement parameters $\left(\AA^{2}\right)$

\begin{tabular}{lllllll}
\hline & $U^{11}$ & $U^{22}$ & $U^{33}$ & $U^{12}$ & $U^{13}$ & $U^{23}$ \\
\hline C1 & $0.0207(6)$ & $0.0240(6)$ & $0.0204(6)$ & $0.0000(5)$ & $0.0020(4)$ & $-0.0009(5)$ \\
C2 & $0.0254(6)$ & $0.0247(6)$ & $0.0254(6)$ & $-0.0045(5)$ & $0.0037(5)$ & $0.0004(5)$ \\
C3 & $0.0293(7)$ & $0.0261(7)$ & $0.0243(6)$ & $-0.0001(5)$ & $0.0037(5)$ & $0.0030(5)$ \\
C4 & $0.0223(6)$ & $0.0311(7)$ & $0.0198(6)$ & $0.0006(5)$ & $0.0029(5)$ & $-0.0023(5)$ \\
C5 & $0.0241(6)$ & $0.0262(6)$ & $0.0246(6)$ & $-0.0044(5)$ & $0.0053(5)$ & $-0.0041(5)$ \\
C6 & $0.0253(6)$ & $0.0223(6)$ & $0.0222(6)$ & $-0.0015(5)$ & $0.0064(5)$ & $-0.0023(5)$ \\
C7 & $0.0315(7)$ & $0.0212(6)$ & $0.0333(7)$ & $-0.0037(5)$ & $0.0041(6)$ & $0.0019(5)$ \\
C8 & $0.0447(9)$ & $0.0336(8)$ & $0.0327(8)$ & $-0.0051(7)$ & $0.0086(7)$ & $0.0086(6)$ \\
C9 & $0.0443(9)$ & $0.0242(7)$ & $0.0498(10)$ & $0.0051(6)$ & $0.0087(7)$ & $-0.0019(7)$ \\
C10 & $0.0439(9)$ & $0.0279(8)$ & $0.0556(11)$ & $-0.0124(7)$ & $-0.0012(8)$ & $0.0062(7)$ \\
C11 & $0.0253(7)$ & $0.0395(8)$ & $0.0322(7)$ & $-0.0019(6)$ & $-0.0021(6)$ & $-0.0082(6)$ \\
O1 & $0.0238(4)$ & $0.0220(4)$ & $0.0260(5)$ & $0.0002(3)$ & $-0.0022(4)$ & $-0.0019(4)$ \\
O2 & $0.0252(5)$ & $0.0360(5)$ & $0.0283(5)$ & $-0.0015(4)$ & $-0.0033(4)$ & $0.0005(4)$ \\
P1 & $0.0288(2)$ & $0.0192(2)$ & $0.0242(2)$ & 0.000 & $-0.00339(18)$ & 0.000 \\
C11 & $0.02605(18)$ & $0.0368(2)$ & $0.0320(2)$ & $0.00652(13)$ & $0.00781(14)$ & $-0.00242(13)$ \\
S1 & $0.02605(18)$ & $0.0368(2)$ & $0.0320(2)$ & $0.00652(13)$ & $0.00781(14)$ & $-0.00242(13)$ \\
& & & & & & \\
\hline
\end{tabular}

Geometric parameters $\left(\AA,{ }^{\circ}\right)$

\begin{tabular}{llll}
\hline $\mathrm{C} 1-\mathrm{C} 2$ & $1.3946(18)$ & $\mathrm{C} 8-\mathrm{H} 8 \mathrm{C}$ & $1.00(2)$ \\
$\mathrm{C} 1-\mathrm{C} 6$ & $1.4086(17)$ & $\mathrm{C} 9-\mathrm{H} 9 \mathrm{~A}$ & $1.00(2)$ \\
$\mathrm{C} 1-\mathrm{O} 1$ & $1.4226(14)$ & $\mathrm{C} 9-\mathrm{H} 9 \mathrm{~B}$ & $1.02(2)$ \\
$\mathrm{C} 2-\mathrm{C} 3$ & $1.3860(18)$ & $\mathrm{C} 9-\mathrm{H} 9 \mathrm{C}$ & $0.994(19)$ \\
$\mathrm{C} 2-\mathrm{H} 2$ & $0.955(16)$ & $\mathrm{C} 10-\mathrm{H} 10 \mathrm{~A}$ & $1.03(2)$ \\
$\mathrm{C} 3-\mathrm{C} 4$ & $1.3979(18)$ & $\mathrm{C} 10-\mathrm{H} 10 \mathrm{~B}$ & $1.02(2)$ \\
$\mathrm{C} 3-\mathrm{H} 3$ & $0.974(17)$ & $\mathrm{C} 10-\mathrm{H} 10 \mathrm{C}$ & $0.96(2)$ \\
$\mathrm{C} 4-\mathrm{O} 2$ & $1.3759(15)$ & $\mathrm{C} 11-\mathrm{O} 2$ & $1.4383(17)$ \\
$\mathrm{C} 4-\mathrm{C} 5$ & $1.4017(19)$ & $\mathrm{C} 11-\mathrm{H} 11 \mathrm{~A}$ & $0.998(18)$ \\
$\mathrm{C} 5-\mathrm{C} 6$ & $1.4088(18)$ & $\mathrm{C} 11-\mathrm{H} 11 \mathrm{~B}$ & $0.990(18)$ \\
$\mathrm{C} 5-\mathrm{H} 5$ & $0.952(16)$ & $\mathrm{C} 11-\mathrm{H} 11 \mathrm{C}$ & $1.009(17)$ \\
$\mathrm{C} 6-\mathrm{C} 7$ & $1.5499(18)$ & $\mathrm{O} 1-\mathrm{P} 1$ & $1.5750(9)$ \\
$\mathrm{C} 7-\mathrm{C} 10$ & $1.5468(19)$ & $\mathrm{P} 1-\mathrm{O} 1^{\mathrm{i}}$ & $1.5750(9)$ \\
$\mathrm{C} 7-\mathrm{C} 8$ & $1.548(2)$ & $\mathrm{P} 1-\mathrm{S} 1^{\mathrm{i}}$ & $1.9791(4)$ \\
$\mathrm{C} 7-\mathrm{C} 9$ & $1.552(2)$ & $\mathrm{P} 1-\mathrm{C} 11^{\mathrm{i}}$ & $1.9791(4)$ \\
$\mathrm{C} 8-\mathrm{H} 8 \mathrm{~A}$ & $1.00(2)$ & $\mathrm{P} 1-\mathrm{C} 11$ & \\
$\mathrm{C} 8-\mathrm{H} 8 \mathrm{~B}$ & $0.948(19)$ & & $10991(4)$ \\
$\mathrm{C} 2-\mathrm{C} 1-\mathrm{C} 6$ & & &
\end{tabular}




\begin{tabular}{|c|c|c|c|}
\hline $\mathrm{C} 2-\mathrm{C} 1-\mathrm{O} 1$ & $119.13(11)$ & $\mathrm{C} 7-\mathrm{C} 9-\mathrm{H} 9 \mathrm{~B}$ & $111.8(11)$ \\
\hline $\mathrm{C} 6-\mathrm{C} 1-\mathrm{O} 1$ & $117.58(11)$ & $\mathrm{H} 9 \mathrm{~A}-\mathrm{C} 9-\mathrm{H} 9 \mathrm{~B}$ & $108.6(16)$ \\
\hline $\mathrm{C} 3-\mathrm{C} 2-\mathrm{C} 1$ & $119.77(12)$ & $\mathrm{C} 7-\mathrm{C} 9-\mathrm{H} 9 \mathrm{C}$ & $112.5(11)$ \\
\hline $\mathrm{C} 3-\mathrm{C} 2-\mathrm{H} 2$ & $120.3(9)$ & $\mathrm{H} 9 \mathrm{~A}-\mathrm{C} 9-\mathrm{H} 9 \mathrm{C}$ & $107.2(15)$ \\
\hline $\mathrm{C} 1-\mathrm{C} 2-\mathrm{H} 2$ & $119.9(9)$ & $\mathrm{H} 9 \mathrm{~B}-\mathrm{C} 9-\mathrm{H} 9 \mathrm{C}$ & $106.6(15)$ \\
\hline $\mathrm{C} 2-\mathrm{C} 3-\mathrm{C} 4$ & $119.11(12)$ & $\mathrm{C} 7-\mathrm{C} 10-\mathrm{H} 10 \mathrm{~A}$ & $111.4(11)$ \\
\hline $\mathrm{C} 2-\mathrm{C} 3-\mathrm{H} 3$ & $121.1(9)$ & $\mathrm{C} 7-\mathrm{C} 10-\mathrm{H} 10 \mathrm{~B}$ & $112.1(11)$ \\
\hline $\mathrm{C} 4-\mathrm{C} 3-\mathrm{H} 3$ & $119.8(9)$ & $\mathrm{H} 10 \mathrm{~A}-\mathrm{C} 10-\mathrm{H} 10 \mathrm{~B}$ & $106.1(16)$ \\
\hline $\mathrm{O} 2-\mathrm{C} 4-\mathrm{C} 3$ & $115.24(12)$ & $\mathrm{C} 7-\mathrm{C} 10-\mathrm{H} 10 \mathrm{C}$ & $108.5(12)$ \\
\hline $\mathrm{O} 2-\mathrm{C} 4-\mathrm{C} 5$ & $124.38(11)$ & $\mathrm{H} 10 \mathrm{~A}-\mathrm{C} 10-\mathrm{H} 10 \mathrm{C}$ & $109.9(16)$ \\
\hline $\mathrm{C} 3-\mathrm{C} 4-\mathrm{C} 5$ & $120.39(11)$ & $\mathrm{H} 10 \mathrm{~B}-\mathrm{C} 10-\mathrm{H} 10 \mathrm{C}$ & $108.8(16)$ \\
\hline $\mathrm{C} 4-\mathrm{C} 5-\mathrm{C} 6$ & $121.99(12)$ & $\mathrm{O} 2-\mathrm{C} 11-\mathrm{H} 11 \mathrm{~A}$ & $111.5(10)$ \\
\hline $\mathrm{C} 4-\mathrm{C} 5-\mathrm{H} 5$ & $117.3(9)$ & $\mathrm{O} 2-\mathrm{C} 11-\mathrm{H} 11 \mathrm{~B}$ & $104.1(10)$ \\
\hline $\mathrm{C} 6-\mathrm{C} 5-\mathrm{H} 5$ & $120.7(9)$ & $\mathrm{H} 11 \mathrm{~A}-\mathrm{C} 11-\mathrm{H} 11 \mathrm{~B}$ & $109.7(14)$ \\
\hline $\mathrm{C} 1-\mathrm{C} 6-\mathrm{C} 5$ & $115.48(11)$ & $\mathrm{O} 2-\mathrm{C} 11-\mathrm{H} 11 \mathrm{C}$ & $111.3(9)$ \\
\hline $\mathrm{C} 1-\mathrm{C} 6-\mathrm{C} 7$ & $123.14(11)$ & $\mathrm{H} 11 \mathrm{~A}-\mathrm{C} 11-\mathrm{H} 11 \mathrm{C}$ & $110.4(14)$ \\
\hline $\mathrm{C} 5-\mathrm{C} 6-\mathrm{C} 7$ & $121.37(11)$ & $\mathrm{H} 11 \mathrm{~B}-\mathrm{C} 11-\mathrm{H} 11 \mathrm{C}$ & $109.7(13)$ \\
\hline $\mathrm{C} 10-\mathrm{C} 7-\mathrm{C} 8$ & $106.40(13)$ & $\mathrm{C} 1-\mathrm{O} 1-\mathrm{P} 1$ & $126.11(8)$ \\
\hline $\mathrm{C} 10-\mathrm{C} 7-\mathrm{C} 6$ & $111.83(11)$ & $\mathrm{C} 4-\mathrm{O} 2-\mathrm{C} 11$ & $117.18(11)$ \\
\hline $\mathrm{C} 8-\mathrm{C} 7-\mathrm{C} 6$ & $110.24(11)$ & $\mathrm{O} 1-\mathrm{P} 1-\mathrm{O} 1^{\mathrm{i}}$ & $97.08(7)$ \\
\hline $\mathrm{C} 10-\mathrm{C} 7-\mathrm{C} 9$ & $107.68(13)$ & $\mathrm{O} 1-\mathrm{P} 1-\mathrm{S} 1^{\mathrm{i}}$ & $111.27(4)$ \\
\hline $\mathrm{C} 8-\mathrm{C} 7-\mathrm{C} 9$ & $110.96(12)$ & $\mathrm{O} 1^{\mathrm{i}}-\mathrm{P} 1-\mathrm{S} 1^{\mathrm{i}}$ & $111.44(3)$ \\
\hline $\mathrm{C} 6-\mathrm{C} 7-\mathrm{C} 9$ & $109.67(12)$ & $\mathrm{O} 1-\mathrm{P} 1-\mathrm{C} 11^{\mathrm{i}}$ & $111.27(4)$ \\
\hline $\mathrm{C} 7-\mathrm{C} 8-\mathrm{H} 8 \mathrm{~A}$ & $109.7(11)$ & $\mathrm{O} 1^{\mathrm{i}}-\mathrm{P} 1-\mathrm{C} 11^{\mathrm{i}}$ & $111.44(3)$ \\
\hline $\mathrm{C} 7-\mathrm{C} 8-\mathrm{H} 8 \mathrm{~B}$ & $112.2(11)$ & $\mathrm{S} 1^{\mathrm{i}}-\mathrm{P} 1-\mathrm{Cl1}^{\mathrm{i}}$ & $0.00(2)$ \\
\hline $\mathrm{H} 8 \mathrm{~A}-\mathrm{C} 8-\mathrm{H} 8 \mathrm{~B}$ & $108.9(16)$ & $\mathrm{O} 1-\mathrm{P} 1-\mathrm{Cl} 1$ & $111.44(3)$ \\
\hline $\mathrm{C} 7-\mathrm{C} 8-\mathrm{H} 8 \mathrm{C}$ & $110.0(12)$ & $\mathrm{O} 1{ }^{\mathrm{i}}-\mathrm{P} 1-\mathrm{C} 11$ & $111.27(4)$ \\
\hline $\mathrm{H} 8 \mathrm{~A}-\mathrm{C} 8-\mathrm{H} 8 \mathrm{C}$ & $106.8(16)$ & $\mathrm{S} 11^{\mathrm{i}}-\mathrm{P} 1-\mathrm{C} 11$ & $113.26(3)$ \\
\hline $\mathrm{H} 8 \mathrm{~B}-\mathrm{C} 8-\mathrm{H} 8 \mathrm{C}$ & $109.0(16)$ & $\mathrm{C} 11^{\mathrm{i}}-\mathrm{P} 1-\mathrm{Cl} 1$ & $113.26(3)$ \\
\hline $\mathrm{C} 6-\mathrm{C} 1-\mathrm{C} 2-\mathrm{C} 3$ & $-0.8(2)$ & $\mathrm{C} 5-\mathrm{C} 6-\mathrm{C} 7-\mathrm{C} 10$ & $2.35(18)$ \\
\hline $\mathrm{O} 1-\mathrm{C} 1-\mathrm{C} 2-\mathrm{C} 3$ & $-178.73(11)$ & $\mathrm{C} 1-\mathrm{C} 6-\mathrm{C} 7-\mathrm{C} 8$ & $-60.70(16)$ \\
\hline $\mathrm{C} 1-\mathrm{C} 2-\mathrm{C} 3-\mathrm{C} 4$ & $1.4(2)$ & $\mathrm{C} 5-\mathrm{C} 6-\mathrm{C} 7-\mathrm{C} 8$ & $120.51(13)$ \\
\hline $\mathrm{C} 2-\mathrm{C} 3-\mathrm{C} 4-\mathrm{O} 2$ & $178.39(11)$ & $\mathrm{C} 1-\mathrm{C} 6-\mathrm{C} 7-\mathrm{C} 9$ & $61.76(16)$ \\
\hline $\mathrm{C} 2-\mathrm{C} 3-\mathrm{C} 4-\mathrm{C} 5$ & $-1.14(19)$ & $\mathrm{C} 5-\mathrm{C} 6-\mathrm{C} 7-\mathrm{C} 9$ & $-117.03(14)$ \\
\hline $\mathrm{O} 2-\mathrm{C} 4-\mathrm{C} 5-\mathrm{C} 6$ & $-179.21(11)$ & $\mathrm{C} 2-\mathrm{C} 1-\mathrm{O} 1-\mathrm{P} 1$ & $-40.07(15)$ \\
\hline $\mathrm{C} 3-\mathrm{C} 4-\mathrm{C} 5-\mathrm{C} 6$ & $0.28(19)$ & $\mathrm{C} 6-\mathrm{C} 1-\mathrm{O} 1-\mathrm{P} 1$ & $141.89(10)$ \\
\hline $\mathrm{C} 2-\mathrm{C} 1-\mathrm{C} 6-\mathrm{C} 5$ & $-0.05(18)$ & $\mathrm{C} 3-\mathrm{C} 4-\mathrm{O} 2-\mathrm{C} 11$ & $-176.65(12)$ \\
\hline $\mathrm{O} 1-\mathrm{C} 1-\mathrm{C} 6-\mathrm{C} 5$ & $177.90(10)$ & $\mathrm{C} 5-\mathrm{C} 4-\mathrm{O} 2-\mathrm{C} 11$ & $2.86(18)$ \\
\hline $\mathrm{C} 2-\mathrm{C} 1-\mathrm{C} 6-\mathrm{C} 7$ & $-178.90(12)$ & $\mathrm{C} 1-\mathrm{O} 1-\mathrm{P} 1-\mathrm{O} 1^{\mathrm{i}}$ & $-155.66(11)$ \\
\hline $\mathrm{O} 1-\mathrm{C} 1-\mathrm{C} 6-\mathrm{C} 7$ & $-0.95(18)$ & $\mathrm{C} 1-\mathrm{O} 1-\mathrm{P} 1-\mathrm{S} 1^{\mathrm{i}}$ & $88.01(9)$ \\
\hline $\mathrm{C} 4-\mathrm{C} 5-\mathrm{C} 6-\mathrm{C} 1$ & $0.31(18)$ & $\mathrm{C} 1-\mathrm{O} 1-\mathrm{P} 1-\mathrm{C} 11^{\mathrm{i}}$ & $88.01(9)$ \\
\hline $\mathrm{C} 4-\mathrm{C} 5-\mathrm{C} 6-\mathrm{C} 7$ & $179.19(12)$ & $\mathrm{C} 1-\mathrm{O} 1-\mathrm{P} 1-\mathrm{Cl} 1$ & $-39.46(10)$ \\
\hline $\mathrm{C} 1-\mathrm{C} 6-\mathrm{C} 7-\mathrm{C} 10$ & $-178.86(13)$ & & \\
\hline
\end{tabular}

Symmetry code: (i) $-x+1, y,-z+1 / 2$. 